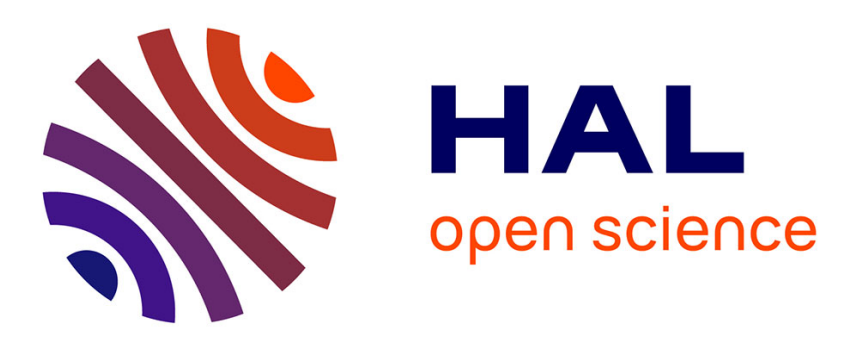

\title{
DISCRIMINATION DES TRAITS PHONÉTIQUES AU TRAVERS DES IMPLANTS COCHLÉAIRES
}

\author{
B. Virole, B. Azema, C. Deleurme
}

\section{To cite this version:}

B. Virole, B. Azema, C. Deleurme. DISCRIMINATION DES TRAITS PHONÉtIQUES AU TRAVERS DES IMPLANTS COCHLÉAIRES. Journal de Physique IV Proceedings, 1992, 02 (C1), pp.C1-197-C1-200. 10.1051/jp4:1992139 . jpa-00251210

\section{HAL Id: jpa-00251210 https://hal.science/jpa-00251210}

Submitted on 1 Jan 1992

HAL is a multi-disciplinary open access archive for the deposit and dissemination of scientific research documents, whether they are published or not. The documents may come from teaching and research institutions in France or abroad, or from public or private research centers.
L'archive ouverte pluridisciplinaire HAL, est destinée au dépôt et à la diffusion de documents scientifiques de niveau recherche, publiés ou non, émanant des établissements d'enseignement et de recherche français ou étrangers, des laboratoires publics ou privés. 


\title{
DISCRIMINATION DES TRAITS PHONETIQUES AU TRAVERS DES IMPLANTS COCHLEAIRES
}

\author{
B. VIROLE, B. AZEMA et C. DELEURME
}

Laboratoire de Recherches Acoustiques, l'Aide Auditive Paris, 230 rue du Fg Saint Honoré, F-75008 Paris, France

\begin{abstract}
RESUME: Nous présentons les résultats de discrimination de 11 patients adultes implantés avec différents systèmes mono et multicanaux. Par comparaison avec les prédictions faites à partir des études sur le codage de la parole dans le nerf auditif par indices neuronaux (1). Nous montrons que si les traits phonétiques de voisement et de continuite sont bien discriminés quels que soient les types d'implants et que la discrimination du trait de nasalité est améliorée dans certains cas, les autres traits phonetiques de tonalité et de compacité ne sont pas catégorisés, même au travers des implants multicanaux.
\end{abstract}

ABSTRACT: The authors present the results of phonetic discrimination in 11 adult patients implanted with variouss mono and multichannel systems. By comparison with predictions based upon studies of the coding of speech in the auditory nerve (1), it is shown that while the phonetic feature of voicing and continuity are well discrimianted regardless of the type of implant and that the discrimination of the feature of nasality is improved in certain cases, the other phonetic feature of tonality and compactness are not categorized, even via multichannel implants.

\section{INTRODUCTION}

Les résultats expérimentaux effectués sur le nerf cochléaire des cobayes et sur modèle électonique montrent qu'il existe dès ce niveau des indices neuronaux pour certaines distinctions phonétiques qui ont une répartition très large en fonction de la fréquence caractéristique (1):

- indices dynamiques pour la distinction entre consonnes continues et discontinues (Cu, DCu) (2).

- indices de périodicité pour la discrimination du Fo (Vo,NVo)

Il est donc théoriquement possible que des implants cochléaires même très simples puissent fournir une information suffisante pour certaines distinctions phonétiques, pouvu que le système d'encodage des formes acoustiques en signaux éléctriques déli- 
vrées par électrodes ne dégrade pas les indices importants (Cf tableau $I$ ). Nous avons essayé de tester cette suggestion en réalisant une analyse phonétique des erreurs réalisées en clinique sur 11 sujets adultes implantés atteints de déficience auditive totale pour lesquels les appareillages audioprothetiques classiques par voie aerienne n'avaient donné aucun résultat appreciable.

\section{ETUDE PHONETIQUE}

Les résultats présentés sur le tableau II représentent pour chaque trait distinctif le gain en taux de catégorisation fourni par l'implant aux résultats obtenus avec la lecture labiale seule. Les valeurs positives attestent que sur le trait considéré 1 implant a fourni des informations supplementaires qui ont permis, avec celles issues de la lecture labiale, une amélioration de la discrimination du trait. Les valeurs négatives correspondent à une interférence négative des informations délivrées par 1 implant avec les stratégies d'identification phonetique étayés sur la lecture labiale. Plus les valeurs sont fortes, en grandeur absolue, et plus elles sont significatives.

L'implant, quel que soit son type, ameliore donc sensiblement les traits de voisement, et de discontinuite, responsables des confusions en lecture labiale seule. Mais il améliore aussi dans une moindre proportion certains autres traits (valeurs $>0$ ). Ce $n$ 'est donc que par l'association de la lecture labiale et des informations fournies par l'implant que la discrimination phonétique s'ameliore nettement. Ceci est cependant éminament variable selon les patients et il existe des traits présentant des valeurs négatives. Dans ce cas, les informations fournies par I'implant sont rentrées en competition avec celles fournies par la lecture labiale et ont abouties à une désorganisation des processus de catégorisation centraux.

Par contre, les indices restitués par la modalité implant seul sont uniquement ceux permettant la distinction Voisé /non Voisé, et Continu/non Continu (Cf tableau III). Le nombre d'électrodes ne semble pas avoir d'effet qualitatif sur la discrimination des traits, mais les résultats quantitatifs attestent que les patients appareillés par multiélectrodes ont des scores généraux d'identification meilleure, grâce à la meilleure discrimination des voyelles. Tout se passe comme si la pluralité des eléctrodes ne permettaient pas, avec implant seul, la géneration d'indices encodant le timbre des phonèmes mais permettait par contre la génération d'indices encodant la périodicité énergétique des voyelles. 


\section{CONCLUSIONS}

Les prédictions expérimentales sur la catégorisation des traits de voisement et de continuité se sont donc trouvés validées sur notre corpus clinique (Tableau IV). Par contre en ce qui concerne les traits de tonalité, de compacité et de nasalité, les prédictions réalisées à partir de la connaissance des indices neuronaux ne se confirment pas. Il semble particulièrement difficile, même avec des systèmes multicanaux de faire percevoir une information distinctive pouvant activer les detecteurs de traits de tonalité. Sur le plan des indices neuronaux décrits par Delgutte, on peut cependant noter que les indices dynamiques (pics des decharges des fibres a l'attaque de transitions) ont été transmis par tous les implants, ainsi que les indices de périodicité (décharge synchrone des fibres avec les fréquences basses caractéristiques du voisement). Cependant les indices d'intensité différentielle, ainsi que les indices de localisation, (traits A/G) n'ont pas été transmis ou n'ont pas pu déclencher la catégorisation.

\section{Référence}

(1) DELGUTTE B. (1984) Codage de la parole dans le nerf auditif, Thèse de Doctorat d'Etat, Université Paris VI.

TABLEAU I. Caractéristiques des Implants

\begin{tabular}{|c|c|c|c|c|}
\hline & : $N b$ & $\begin{array}{l}\text { electrodes } \\
\text { actives }\end{array}$ & $\begin{array}{l}: \\
:\end{array}$ & codage \\
\hline$:$ & : & & : & \\
\hline :NUCLEUS & : & (11) & : & Numérique $F O / F 1 / F 2$ \\
\hline :SYMBION & $:$ & (4) & : & Anlogique 4 bandes \\
\hline :HORTMAN & $:$ & (8) & : & Numérique (vocoder) \\
\hline : IMPContour & & (1) & : & Analogique \\
\hline
\end{tabular}


TABLEAU II. Gain en categorisation fourni par I'implant a la discrimination phonétique en lecture labiale

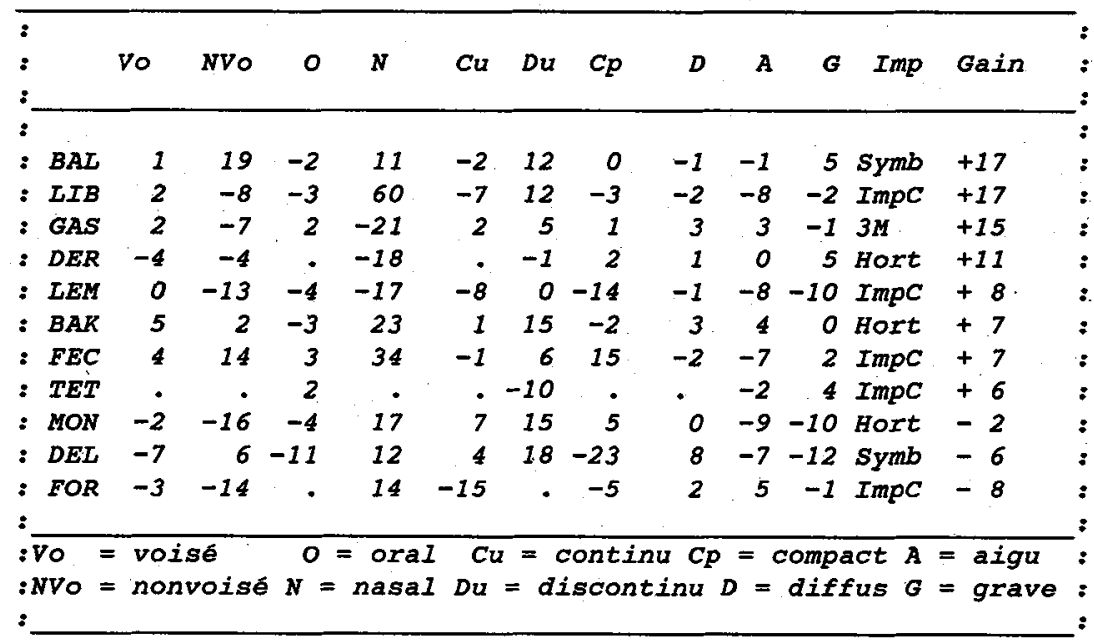

TABLEAO III. Taux d'erreurs de discrimination sur les traits phonétiques (Ecarts aux probabilités d'erreurs au hasard). Modalité implant seul.

\begin{tabular}{|c|c|c|c|c|c|c|c|c|c|c|c|}
\hline : & Vo & NVO & $o$ & $N$ & $\mathrm{Cu}$ & $D u$ & $C p$ & $D$ & A & $G$ & Implant \\
\hline$:$ & & & & & & & & & & & 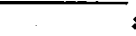 \\
\hline : SID & 8 & 15 & 8 & -20 & 6 & 12 & -23 & 7 & -8 & -12 & Nucleus \\
\hline$: B A L$ & 2 & -6 & -2 & -29 & -8 & -6 & -18 & -7 & -18 & -9 & symbion \\
\hline : MON & 10 & 3 & 12 & -24 & -1 & • & -4 & • & 7 & 8 & Hortman \\
\hline$: M I C$ & 11 & 1 & 0 & -17 & 3 & 5 & 7 & 1 & -11 & 0 & symbion \\
\hline : IOU & 15 & 3 & 7 & -36 & 5 & -16 & -27 & 3 & 3 & -11 & symbion \\
\hline
\end{tabular}

TABLEAU IV. Moyenne des écarts aux probabilités d'erreurs au hasard sur les traits phonétiques.

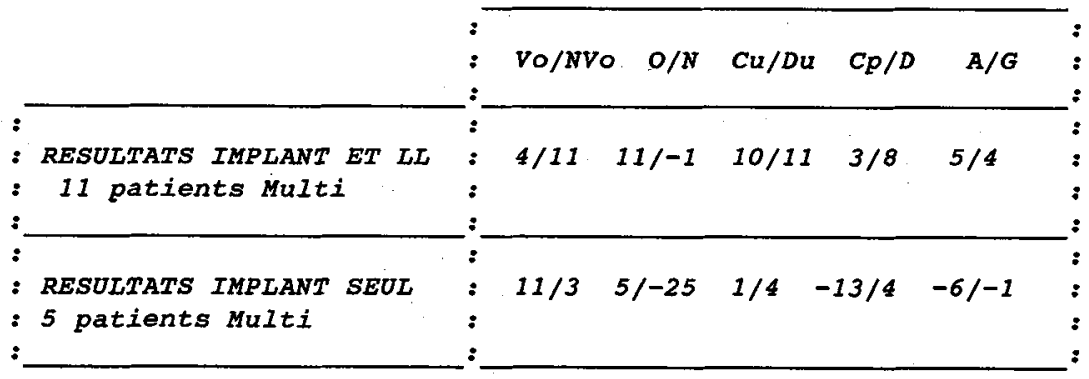

